\title{
A projection-type method for generalized variational inequalities with dual solutions
}

\author{
Ming Zhu, Guo-Ji Tang* \\ School of Science, Guangxi University for Nationalities, Nanning, Guangxi 530006, P. R. China.
}

Communicated by S. S. Chang

\begin{abstract}
In this paper, a new projection-type method for generalized variational inequalities is introduced in Euclidean spaces. Under the assumption that the dual variational inequality has a solution, we show that the proposed method is well-defined and prove that the sequence generated by the proposed method is convergent to a solution, where the condition is strictly weaker than the pseudomonotonicity of the mapping used by some authors. We provide an example to support our results. Compared with the recent works of Li and He [F.-L. Li, Y.-R. He, J. Comput. Appl. Math., 228 (2009), 212-218], and Fang and He [C.-J. Fang, Y.-R. He, Appl. Math. Comput., 217 (2011), 9543-9551], condition (A3) is removed. Moreover, the results presented in this paper also generalize and improve some known results given in other literature. (c)2017 All rights reserved.
\end{abstract}

Keywords: Projection method, generalized variational inequality, dual variational inequality. 2010 MSC: $90 \mathrm{C} 33$.

\section{Introduction}

Let $C$ be a nonempty, closed, and convex subset of $\mathbb{R}^{n}$ and $F: C \rightrightarrows \mathbb{R}^{n}$ be a set-valued mapping. Denote the usual inner product and norm in $\mathbb{R}^{n}$ by $\langle\cdot, \cdot\rangle$ and norm $\|\cdot\|$, respectively. We consider the generalized variational inequality (in short $\operatorname{GVI}(F, C)$ ): find $x^{*} \in C$ and $\xi \in F\left(x^{*}\right)$ such that

$$
\left\langle\xi, y-x^{*}\right\rangle \geqslant 0, \quad \forall y \in C .
$$

For convenience, we denote by $\operatorname{SOL}(F, C)$ the set of solutions of $\mathrm{GVI}(\mathrm{F}, \mathrm{C})$. If $\mathrm{F}$ is single-valued, then $\mathrm{GVI}(\mathrm{F}, \mathrm{C})$ reduces to the variational inequality (in short $\mathrm{VI}(\mathrm{F}, \mathrm{C})$ ), i.e., find $x^{*} \in \mathrm{C}$ such that

$$
\left\langle\mathrm{F}\left(\mathrm{x}^{*}\right), \mathrm{y}-\mathrm{x}^{*}\right\rangle \geqslant 0, \quad \forall \mathrm{y} \in \mathrm{C} .
$$

It is well-known that the solution of $\operatorname{GVI}(\mathrm{F}, \mathrm{C})$ is closely related with that of the dual variational inequality problem (in short DVI $(F, C)$ ) of finding $x^{*} \in C$ such that

$$
\forall y \in C \text { and } \forall \eta \in F(y), \quad\left\langle\eta, y-x^{*}\right\rangle \geqslant 0 .
$$

\footnotetext{
*Corresponding author

Email address: guojvtang@126.com, guojvtang@hotmail.com (Guo-ji Tang)
}

doi:10.22436/jnsa.010.09.23 
Such an element $x^{*} \in C$ satisfying (1.1) is called a solution of DVI $(F, C)$, or a dual solution of GVI $(F, C)$. The set of all solutions of $\mathrm{DVI}(\mathrm{F}, \mathrm{C})$ is denoted by $\mathrm{SOL}_{\mathrm{D}}(\mathrm{F}, \mathrm{C})$.

In the theory of variational inequalities, it is an important part to devise some algorithms to find an approximate solution of variational inequalities. In this paper, we will focus on the topic of projection-type methods. Thus, we should recall some development process of projection-type methods. The simplest version among projection-type methods for solving $\mathrm{VI}(\mathrm{F}, \mathrm{C})$ is the Picard iteration, which depends on its equivalent fixed point reformulation $x=P_{C}(x-\mu F(x))$. In order to get the convergence, Picard iteration scheme requires very strong conditions, i.e., the strong monotonicity and Lipschitz continuity of the mapping F. In 1976, Korpelevič [10] constructed a double projection method such that the strong monotonicity of $F$ is relaxed to its monotonicity. In 1997, Iusem and Svaiter [8] introduced a variant of Korpelevich's method by using Armijo linear search procedure such that the Lipschitz continuity of $F$ is further relaxed to the continuity. Then He [5] constructed a new double projection method by introducing another Armijo type linear search different from that of Iusem and Svaiter [8], and analyzed the rate of convergence. Recently, based on the crucial points of convergence proof of related projection methods, Ye and He [17] proposed a double projection method for $\operatorname{VI}(\mathrm{F}, \mathrm{C})$. They require that dual variational inequality has a solution, which is weaker than pseudomonotonicity of the mapping. For more related works, we refer the readers to the references $[6,14-16]$.

For $\operatorname{GVI}(\mathrm{F}, \mathrm{C})$, the appearance of set-valued mapping causes solving $\mathrm{GVI}(\mathrm{F}, \mathrm{C})$ and analyzing the convergence of the methods to become more complicated. As mentioned by Iusem and Perez [7], the point-to-point mappings in $\mathrm{VI}(\mathrm{F}, \mathrm{C})$ can not be automatically extended to point-to-set mappings. In algorithms for solving $\mathrm{VI}(\mathrm{F}, \mathrm{C})$, if we just replace $\mathrm{F}(\mathrm{x})$ by $u \in F(x)$, then the algorithm might be unsuccessful. They also provide an example to support his viewpoint in Sect. 2 of [7]. Thus, it is interesting to deal with the algorithms for $\mathrm{GVI}(\mathrm{F}, \mathrm{C})$.

If $T$ is maximal monotone, Iusem and Perez [7] suggested an extragradient method to solve GVI(F, C). In 2009, $\mathrm{Li}$ and He [11] devised a projection type method to solve GVI(F, C), where for taking an element in $F\left(x_{i}\right)$, they have to solve another single-valued variational inequality. For more details, the reader may refer to the references [7, 11] (see, for example, (35) and (36) of [7], and (2.1) of [11]). Therefore the price at each iteration is somewhat expensive. To overcome this difficulty, Fang and He [2] modified the algorithms and it is arbitrary to take an element in $\mathrm{F}\left(\mathrm{x}_{i}\right)$. Both $\mathrm{Li}$ and $\mathrm{He}$ [11] and Fang and He [2], in order to analyze the convergence of the algorithms, adopted the same assumptions as follows:

(A1) $\mathrm{SOL}(\mathrm{F}, \mathrm{C})$ is nonempty;

(A2) $\mathrm{F}$ is continuous with nonempty compact convex values;

(A3) for each $x \in \operatorname{SOL}(F, C)$, it holds that

$$
\langle\eta, y-x\rangle \geqslant 0, \quad \forall y \in C \text { and } \forall \eta \in F(y) .
$$

It is easy to see that if $F$ is pseudomonotone (see Definition 2.1), then condition (A3) is true. The singlevalued version of condition (A3) is also used in literatures, see, for example [13]. However, since we do not know all elements of $\mathrm{SOL}(\mathrm{F}, \mathrm{C})$ in advance (otherwise there is nothing to do), it is not easy to check condition (A3). On the other hand, there are many applications from reality such that the mapping $\mathrm{F}$ in the model GVI $(F, C)$ is not pseudomonotone, see for example $[3,9,12]$ and the references therein. A natural problem is whether there is a more efficient condition that can replace (A3), or how to weaken condition (A3), even the best is whether condition (A3) can be removed.

Motivated and inspired by the research work mentioned above, in this paper, we suggest a new projection method for GVI $(F, C)$ in Euclidean spaces. Under very mild assumptions, we prove that the sequence generated by the proposed method is globally convergent to a solution of the problem. In some extent, we remove condition (A3) and do not need to add other conditions to get the convergence of the proposed algorithm. 


\section{Preliminaries}

In this section, we shall recall some notations, definitions, and other results, which will be used in the sequel. The following definition regarding inner point is taken from $[4,9]$. For a given set $C \subset \mathbb{R}^{n}$, its convex hull is denoted by $\operatorname{conv}(C)$. For any $x, y \in \mathbb{R}^{n}$, we set

$$
[x, y]:=\operatorname{conv}\{x, y\} \text {. }
$$

Analogously, we can define the segments $(x, y],[x, y)$, and $(x, y)$. A point $t \in \mathbb{R}^{n}$ is said to be perpendicular to $C$ if $\langle t, x\rangle$ is constant for all $x \in C$. The set of perpendicular elements to $C$ is denoted by $C^{P}$. $A$ point $y \in C$ is said to be an inner point of $C$ if, for any $t \in \mathbb{R}^{n} \backslash\{0\}$, the following implication holds:

$$
\langle\mathrm{t}, \mathrm{x}\rangle \leqslant\langle\mathrm{t}, \mathrm{y}\rangle, \quad \forall x \in \mathrm{C} \Rightarrow \mathrm{t} \in \mathrm{C}^{\mathrm{P}} .
$$

The set of inner points of $C$ is denoted by inn $(C)$.

Definition 2.1. A set-valued operator $F: \mathbb{R}^{n} \rightrightarrows \mathbb{R}^{n}$ is said to be

(i) monotone if we have

$$
\langle u-v, x-y\rangle \geqslant 0, \quad \forall x, y \in \mathbb{R}^{n} \text { and } \forall u \in F(x), v \in F(y) ;
$$

(ii) maximal monotone if it is monotone and not properly contained in another monotone operator in the sense of their graphs;

(iii) pseudomonotone if for each pair of points $x, y \in \mathbb{R}^{n}$, and for all $u \in F(x), v \in F(y)$, we have

$$
\langle u, y-x\rangle \geqslant 0 \text { implies }\langle v, y-x\rangle \geqslant 0
$$

(iv) quasimonotone if for each pair of points $x, y \in \mathbb{R}^{n}$, and for all $u \in F(x), v \in F(y)$, we have

$$
\langle u, y-x\rangle>0 \text { implies }\langle v, y-x\rangle \geqslant 0
$$

(v) strictly quasimonotone if it is quasimonotone and for every distinct $x, y \in \mathbb{R}^{n}$, there exists $z \in(x, y)$ such that

$$
\langle\eta, x-y\rangle \neq 0, \quad \forall \eta \in F(z)
$$

(vi) semistrictly quasimonotone if it is quasimonotone and for every distinct $x, y \in \mathbb{R}^{n}$, the relation

$$
\langle\zeta, x-y\rangle>0, \quad \text { for some } \zeta \in F(y),
$$

implies

$$
\langle\eta, x-y\rangle>0, \quad \text { for some } z \in\left(\frac{x+y}{2}, x\right) \text { and } \forall \eta \in F(z) \text {. }
$$

Remark 2.2. The notions of monotonicity, maximal monotonicity, pseudomonotonicity, and quasimonotonicity are well-known. The notions of strict quasimonotonicity and semistrict quasimonotonicity for the single-valued mapping is introduced by Hadjisavvas and Schaible [3], while the analogues for the set-valued mapping are taken from the work of Konnov [9].

Remark 2.3. Various (generalized) monotonicity in Definition 2.1 has the following implication relations:

(a) Maximal monotonicity $\Rightarrow$ Monotonicity $\Rightarrow$ Pseudomonotonicity $\Rightarrow$ Semistrict quasimonotonicity $\Rightarrow$ Quasimonotonicity;

(b) Strict quasimonotonicity $\Rightarrow$ Semistrict quasimonotonicity.

Definition 2.4. A set-valued operator $F: \mathbb{R}^{n} \rightrightarrows \mathbb{R}^{n}$ is said to be

(i) upper semicontinuous at $x \in \mathbb{R}^{n}$ if for every open set $V$ containing $F(x)$, there is an open set $U$ containing $x$ such that $\mathrm{F}(\mathrm{y}) \subset \mathrm{V}$ for all $\mathrm{y} \in \mathrm{U}$; 
(ii) lower semicontinuous at $x \in \mathbb{R}^{n}$ if for any sequence $\left\{x_{k}\right\}$ converging to $x$ and each $y \in F(x)$, there exists a sequence $\left\{y_{k}\right\}$ with $y_{k} \in F\left(x_{k}\right)$, converging to $y$;

(iii) continuous at $x \in \mathbb{R}^{n}$ if it is both upper semicontinuous and lower semicontinuous at $x$;

(iv) upper semicontinuous (resp. lower semicontinuous, continuous) if it is upper semicontinuous (resp. lower semicontinuous, continuous) at each $x \in \mathbb{R}^{n}$;

(v) hemicontinuous if $F$ is upper semicontinuous from line segment of $\mathbb{R}^{n}$ to $\mathbb{R}^{n}$.

For a nonempty, closed, and convex set $C$ in $\mathbb{R}^{n}$ and a point $x \in \mathbb{R}^{n}$, the orthogonal projection of $x$ onto $\mathrm{C}$, denoted by $\mathrm{P}_{\mathrm{C}}(\mathrm{x})$, is defined as

$$
P_{C}(x):=\arg \min \{\|y-x\|: y \in C\} .
$$

Lemma 2.5 ([5, Lemma 2.4]). Let $\mathrm{X}$ be a nonempty, closed, and convex set, $\bar{x}=\mathrm{P}_{\mathrm{X}}(\mathrm{x})$ and $\mathrm{x}^{*} \in \mathrm{X}$. Then one has

$$
\left\|\bar{x}-x^{*}\right\|^{2} \leqslant\left\|x-x^{*}\right\|^{2}-\|x-\bar{x}\|^{2} .
$$

Let $\mu>0$ be a parameter.

Proposition 2.6. $x \in \mathrm{C}$ and $\xi \in \mathrm{F}(\mathrm{x})$ solves $\mathrm{GVI}(\mathrm{F}, \mathrm{C})$ if and only if

$$
r_{\mu}(x, \xi):=x-P_{C}(x-\mu \xi)=0 .
$$

Lemma 2.7 ([11, Lemma 2.3]). For any $x \in \mathrm{C}$ and $\xi \in \mathrm{F}(\mathrm{x})$, one has

$$
\left\langle\xi, r_{\mu}(x, \xi)\right\rangle \geqslant \mu^{-1}\left\|r_{\mu}(x, \xi)\right\|^{2} .
$$

Unless specified otherwise, in this paper we will adopt the following assumptions.

(H1) The set of solutions for $\mathrm{DVI}(\mathrm{F}, \mathrm{C})$ is nonempty, i.e., $\mathrm{SOL}_{\mathrm{D}}(\mathrm{F}, \mathrm{C}) \neq \emptyset$;

(H2) $\mathrm{F}: \mathbb{R}^{\mathrm{n}} \rightrightarrows \mathbb{R}^{\mathrm{n}}$ is continuous with nonempty and compact values.

Regarding assumption (H1), in order to show its reasonability, we should give some sufficient conditions to ensure that $(\mathrm{H} 1)$ holds. Now we need the notions of the trivial solution set and the nontrivial solution set of $\operatorname{GVI}(\mathrm{F}, \mathrm{C})$, that is,

$$
\begin{aligned}
& \operatorname{SOL}_{\mathrm{T}}(\mathrm{F}, \mathrm{C}):=\left\{\chi^{*} \in \mathrm{C} \mid \text { there is } \xi \in \mathrm{F}\left(x^{*}\right) \text { such that }\left\langle\xi, y-\chi^{*}\right\rangle=0, \quad \forall \mathrm{y} \in \mathrm{C}\right\}, \\
& \operatorname{SOL}_{\mathrm{N}}(\mathrm{F}, \mathrm{C}):=\operatorname{SOL}(\mathrm{F}, \mathrm{C}) \backslash \mathrm{SOL}_{\mathrm{T}}(\mathrm{F}, \mathrm{C}) .
\end{aligned}
$$

Theorem 2.8. If one of the following conditions holds:

(i) $\mathrm{F}$ is pseudomonotone on $\mathrm{C}$ and $\operatorname{SOL}(\mathrm{F}, \mathrm{C}) \neq \emptyset$;

(ii) $\mathrm{F}$ is quasimonotone on $\mathrm{C}$ and $\mathrm{SOL}_{\mathrm{N}}(\mathrm{F}, \mathrm{C}) \neq \emptyset$;

(iii) condition (A3) is true,

then $\mathrm{SOL}_{\mathrm{D}}(\mathrm{F}, \mathrm{C})$ is nonempty.

Proof.

(i) This is a direct consequence of the pseudomonotonicity of $\mathrm{F}$ and $\operatorname{SOL}(\mathrm{F}, \mathrm{C}) \neq \emptyset$.

(ii) Combining the definitions of $\mathrm{SOL}_{\mathrm{N}}(\mathrm{F}, \mathrm{C})$ and the quasimonotonicity of $\mathrm{F}$, we easily get it.

(iii) It is trivial.

The following two results are taken from Theorems 4.1 and 4.2 of Konnov [9]. 
Theorem 2.9. Let $\mathrm{C}$ is a nonempty, bounded, closed, and convex subset of $\mathbb{R}^{\mathrm{n}}$ with inn $(\mathrm{C}) \neq \emptyset$. Let $\mathrm{F}$ be a hemicontinuous, semistrictly quasimonotone mapping with nonempty and compact values. Then $S O L_{\mathrm{D}}(\mathrm{F}, \mathrm{C})$ is nonempty.

Theorem 2.10. Let $\mathrm{C}$ is a nonempty, closed and convex subset of $\mathbb{R}^{n}$ with inn $(\mathrm{C}) \neq \emptyset$. Let $\mathrm{F}$ be a hemicontinuous, semistrictly quasimonotone mapping with nonempty and compact values. Suppose that there exists a compact subset $\mathrm{W}$ of $\mathbb{R}^{\mathrm{n}}$ and a point $w^{0} \in \mathrm{W} \cap \operatorname{inn}(\mathrm{C})$ such that, for every $\mathrm{x} \in \mathrm{C} \backslash \mathrm{W}$, there exists $\mathrm{g} \in \mathrm{F}\left(w^{0}\right)$ such that

$$
\left\langle g, w^{0}-x\right\rangle<0
$$

Then $\mathrm{SOL}_{\mathrm{D}}(\mathrm{F}, \mathrm{C})$ is nonempty.

\section{Algorithm}

In this section, we will state our algorithm and show the well-definedness of the proposed algorithm.

\section{Algorithm 3.1.}

Initialization: Choose an initial point $\chi^{0} \in \mathrm{C}$ and parameters $\gamma, \sigma \in(0,1)$ and $\mu \in\left(0, \frac{1}{\sigma}\right)$. Set $k:=0$.

Iteration Step: Given $x^{k} \in C$, if $r_{\mu}\left(x^{k}, \xi\right)=0$ for some $\xi \in F\left(x^{k}\right)$, then stop. Otherwise, take $\xi^{k} \in F\left(x^{k}\right)$ arbitrarily and compute

$$
\begin{gathered}
m_{k}=\min \left\{k \in \mathbb{N}_{+} \mid \inf _{y \in F\left(x^{k}-\gamma^{k} r_{\mu}\left(x^{k}, \xi^{k}\right)\right)}\left\langle\xi^{k}-y, r_{\mu}\left(x^{k}, \xi^{k}\right)\right\rangle \leqslant \sigma\left\|r_{\mu}\left(x^{k}, \xi^{k}\right)\right\|^{2}\right\}, \\
\eta_{k}=\gamma^{m_{k},}
\end{gathered}
$$

and

$$
z^{k}=x^{k}-\eta_{k} r_{\mu}\left(x^{k}, \xi^{k}\right)
$$

Compute

$$
x^{k+1}=P_{C \cap \tilde{H}_{k}}\left(x^{k}\right),
$$

where $\tilde{H}_{k}=\cap_{j=0}^{k} H_{j}$ with $H_{j}:=\left\{v \in \mathbb{R}^{n} \mid h_{j}(v) \leqslant 0\right\}$ is a halfspace dependent on the function

$$
h_{j}(v):=\sup _{\xi \in F\left(z^{j}\right)}\left\langle\xi, v-z^{j}\right\rangle .
$$

Let $k:=k+1$ and return to the Iteration Step.

Remark 3.2.

(i) If F is single-valued, then Algorithm 3.1 reduces to Algorithm 2.1 of Ye and He [17].

(ii) Compared with Algorithm 1 of Fang and He [2], in the projection step (3.2) of our algorithm, the constraint set $\tilde{H}_{k}$ is the intersection of existing halfspaces $H_{j}$ for $j$ from 0 to $k$. This leads to accelerate the convergence of the proposed algorithm in some extent. In addition, this will not cause expensive computation burden, since $\mathrm{H}_{j}$ for $j$ from 0 to $k-1$ have achieved in the previous iteration.

(iii) Compared with Algorithm 1 of $\mathrm{Li}$ and $\mathrm{He}[11], \xi^{k}$ can be taken arbitrarily in our algorithm, while $\xi^{k}$ is selected by solving a single-valued inequality in [11]. It seems that it will cause expensive computation. Moreover, there is other difference between our algorithm and Li and He's algorithm, for example, Armijo-type linesearch procedure and projection step.

In what follows, we should show the well-definedness of Algorithm 3.1. 
Lemma 3.3 ([2, Proposition 2.2]). If $r_{\mu}\left(x^{k}, \xi\right)=0$ for some $\xi \in F\left(x^{k}\right)$, then $x^{k} \in S O L(F, C)$. Otherwise, the linesearch (3.1) in Algorithm 3.1 is well defined.

Lemma 3.4. Let the function $\mathrm{h}_{\mathrm{j}}$ be defined by (3.3) and $\mathrm{x}^{\mathrm{k}}$ be given in Algorithm 3.1. If $S O L_{\mathrm{D}}(\mathrm{F}, \mathrm{C}) \neq \emptyset$, then one has

$$
h_{k}\left(x^{k}\right) \geqslant \eta_{k}\left(\mu^{-1}-\sigma\right)\left\|r_{\mu}\left(x^{k}, \xi^{k}\right)\right\|^{2} .
$$

If $x^{*} \in S O L_{\mathrm{D}}(\mathrm{F}, \mathrm{C})$, then

$$
h_{k}\left(x^{*}\right) \leqslant 0
$$

Proof. Since $z^{k}=x^{k}-\eta_{k} r_{\mu}\left(x^{k}, \xi^{k}\right)$, we have

$$
\begin{aligned}
h_{k}\left(x^{k}\right)=\sup _{\xi \in F\left(z^{k}\right)}\left\langle\xi, x^{k}-z^{k}\right\rangle=\eta_{k} \sup _{\xi \in F\left(z^{k}\right)}\left\langle\xi, r_{\mu}\left(x^{k}, \xi^{k}\right)\right\rangle & \geqslant \eta_{k}\left\langle\xi^{k}, r_{\mu}\left(x^{k}, \xi^{k}\right)\right\rangle-\sigma \eta_{k}\left\|r_{\mu}\left(x^{k}, \xi^{k}\right)\right\|^{2} \\
& \geqslant \eta_{k}\left(\mu^{-1}-\sigma\right)\left\|r_{\mu}\left(x^{k}, \xi^{k}\right)\right\|^{2},
\end{aligned}
$$

where the first inequality follows from (3.1) and the second inequality follows from (2.1). If $x^{*} \in$ $\mathrm{SOL}_{\mathrm{D}}(\mathrm{F}, \mathrm{C})$, then one has

$$
h_{k}\left(x^{*}\right)=\sup _{\xi \in F\left(z^{k}\right)}\left\langle\xi, x^{*}-z^{k}\right\rangle \leqslant 0
$$

This completes the proof.

Lemma 3.5. The projection step (3.2) in Algorithm 3.1 is well-defined.

Proof. To this end, it is sufficient to prove that $\mathrm{C} \cap \tilde{\mathrm{H}}_{\mathrm{k}}$ is nonempty, closed, and convex. By Lemma 3.4 and assumption (H1), we have that

$$
\emptyset \neq \mathrm{SOL}_{\mathrm{D}}(\mathrm{F}, \mathrm{C}) \subseteq \mathrm{H}_{\mathrm{j}}
$$

for $j=0, \cdots, k$. Obviously, $\operatorname{SOL}_{D}(F, C) \subseteq C$. So the set $C \cap \tilde{H}_{k}$ is nonempty. It is easy to see that it is closed and convex. This completes the proof.

Remark 3.6. From Lemmas 3.3 and 3.5, we conclude that Algorithm 3.1 is well-defined under the assumption (H1).

\section{Convergence analysis}

Lemma 4.1 ([11, Lemma 2.2]). The function $h_{j}$ defined by (3.3) is Lipschitz with a constant $M:=\sup _{\xi \in F\left(z^{k}\right)}\|\xi\|$ on $\mathbb{R}^{\mathrm{n}}$.

Lemma 4.2 ([5, Lemma 2.3]). Let $\mathrm{C}$ be a closed and convex subset of $\mathbb{R}^{n}, \mathrm{~h}$ be a real-valued function on $\mathbb{R}^{n}$, and $\mathrm{K}$ be the set $\{\mathrm{x} \in \mathrm{C}: \mathrm{h}(\mathrm{x}) \leqslant 0\}$. If $\mathrm{K}$ is nonempty and $\mathrm{h}$ is Lipschitz continuous on $\mathrm{C}$ with modulus $\alpha>0$, then

$$
\operatorname{dist}(x, K) \geqslant \alpha^{-1} \max \{h(x), 0\}, \quad \forall x \in C,
$$

where $\operatorname{dist}(\mathrm{x}, \mathrm{K})$ denotes the distance from $\mathrm{x}$ to $\mathrm{K}$.

Lemma 4.3. Let $\left\{x^{k}\right\}$ be an infinite sequence generated by Algorithm 3.1 and $\tilde{x}$ be any cluster point of $\left\{x^{k}\right\}$. Then one has $\tilde{x} \in \cap_{k=1}^{\infty} H_{k}$.

Proof. To this end, for any given nonnegative integer $l$, we will show that $\tilde{x} \in H_{l}$. Since $\tilde{x}$ is a cluster point of $\left\{x^{k}\right\}$, there is a subsequence $\left\{x^{k_{i}}\right\}$ of $\left\{x^{k}\right\}$, such that

$$
\lim _{i \rightarrow \infty} x^{k_{i}}=\tilde{x}
$$

According to the definitions of $x^{k_{i}}=P_{C \cap \tilde{H}_{k_{i}-1}}\left(x^{k_{i}-1}\right)$ and $\tilde{H}_{k_{i}-1}=\cap_{j=0}^{j=k_{i}-1} H_{j}$, we have $x^{k_{i}} \in H_{l}$ for all $i \geqslant l+1$. Combining the closedness of $H_{l}$ and (4.2), we have $\tilde{x} \in H_{l}$. This completes the proof. 
We now present the convergence result of Algorithm 3.1.

Theorem 4.4. Under the assumptions $(\mathrm{H} 1)$ and $(\mathrm{H} 2)$, if Algorithm 3.1 terminates at the $\mathrm{k}$-th iteration, then $\mathrm{x}^{\mathrm{k}}$ is a solution of $G V I(F, C)$. Otherwise, the infinite sequence $\left\{x^{k}\right\}$ generated by Algorithm 3.1 is convergent to a solution of $\operatorname{GVI}(\mathrm{F}, \mathrm{C})$.

Proof. The first conclusion follows from Lemma 3.3. Now let $\left\{x^{k}\right\}$ be the infinite sequence generated by Algorithm 3.1. According to the stop criterion, one knows that $r_{\mu}\left(x^{k}, \xi^{k}\right) \neq 0$ for each $k$ and all $\xi^{k} \in F\left(x^{k}\right)$. By $x^{k+1}=P_{C \cap \tilde{H}_{k}}\left(x^{k}\right)$, applying Lemma 2.5, one has that for each $x^{*} \in \cap_{k=0}^{\infty}\left(H_{k} \cap C\right)$,

$$
\left\|x^{k+1}-x^{*}\right\|^{2} \leqslant\left\|x^{k}-x^{*}\right\|^{2}-\left\|x^{k+1}-x^{k}\right\|^{2}=\left\|x^{k}-x^{*}\right\|^{2}-\operatorname{dist}^{2}\left(x^{k}, C \cap \tilde{H}_{k}\right) .
$$

It is easy to see that the sequence $\left\{\left\|x^{k}-x^{*}\right\|\right\}$ is nonincreasing and bounded below. Hence $\lim _{k \rightarrow \infty} \| x^{k}-$ $x^{*} \|$ exists. This implies that $\left\{x^{k}\right\}$ is bounded and

$$
\lim _{k \rightarrow \infty} \operatorname{dist}\left(x^{k}, C \cap \tilde{H}_{k}\right)=0 .
$$

By the definition of $\tilde{H}_{k}$, we have $\tilde{H}_{k} \subset H_{k}$ for all $k$. Thus, it follows that

$$
\operatorname{dist}\left(x^{k}, C \cap \tilde{H}_{k}\right) \geqslant \operatorname{dist}\left(x^{k}, C \cap H_{k}\right)
$$

and so

$$
\lim _{k \rightarrow \infty} \operatorname{dist}\left(x^{k}, \mathrm{C} \cap \mathrm{H}_{k}\right)=0 .
$$

By the boundedness of $\left\{x^{k}\right\}$, there is a convergent subsequence $\left\{x^{k_{i}}\right\}$ converging to $\bar{x}$. Since $F$ is an upper semicontinuous mapping with compact values, it follows from Proposition 3.11 of [1] that $\left\{\mathrm{F}\left(\mathrm{x}^{\mathrm{k}}\right) \mid \mathrm{k} \in \mathbb{N}\right\}$ is a bounded set, and so the sequences $\left\{\xi^{k}\right\},\left\{r_{\mu}\left(x^{k}, \xi^{k}\right)\right\}$, and $\left\{z^{k}\right\}$ are bounded. Thus, akin to the previous argument, $\left\{F\left(z^{k}\right) \mid k \in \mathbb{N}\right\}$ is a bounded set, i.e., there is $M>0$ such that

$$
\sup _{\xi \in F\left(z^{k}\right)}\|\xi\| \leqslant M, \quad \text { for all } k .
$$

It follows from (4.1) and (3.4) that

$$
\operatorname{dist}\left(x^{k}, C \cap H_{k}\right) \geqslant M^{-1} h_{k}\left(x^{k}\right) \geqslant M^{-1} \eta_{k}\left(\mu^{-1}-\sigma\right)\left\|r_{\mu}\left(x^{k}, \xi^{k}\right)\right\|^{2} .
$$

Thus, by (4.4) and (4.5), we have

$$
\lim _{k \rightarrow \infty} \eta_{k}\left\|r_{\mu}\left(x^{k}, \xi^{k}\right)\right\|^{2}=0
$$

We now consider two possible cases.

Case 1: Suppose that $\eta_{k} \nrightarrow 0$ as $k \rightarrow \infty$. Then there is a subsequence of $\left\{\eta_{k}\right\}$, still denoted by $\left\{\eta_{k}\right\}$, and some $\eta>0$ such that $\eta_{k}>\eta$ for all k. Thus, it follows from (4.6) that $\lim _{k \rightarrow \infty}\left\|r_{\mu}\left(x^{k}, \xi^{k}\right)\right\|=0$. Since $r_{\mu}(\cdot, \cdot)$ is continuous and the sequences $\left\{x^{k}\right\}$ and $\left\{\xi^{k}\right\}$ are bounded, there is a cluster point $(\hat{x}, \hat{\xi})$ of $\left\{\left(x^{k}, \xi^{k}\right)\right\}$ such that $r_{\mu}(\hat{x}, \hat{\xi})=0$. This implies that $\hat{x}$ is a solution of $G V I(F, C)$ by Proposition 2.6. By Lemma 4.3, we have $\hat{x} \in \cap_{k=1}^{\infty}\left(H_{k} \cap \operatorname{SOL}(F, C)\right)$. Replacing $x^{*}$ by $\hat{x}$ in (4.3), we have that the sequence $\left\{\left\|x^{k}-\hat{x}\right\|\right\}$ is nonincreasing and hence convergent. Since $\hat{x}$ is a cluster point of $\left\{x^{k}\right\}$, some subsequence of $\left\{\left\|x^{k}-\hat{x}\right\|\right\}$ is convergent to zero. This shows that the whole sequence $\left\{\left\|x^{k}-\hat{x}\right\|\right\}$ is also convergent to zero, and hence $\lim _{k \rightarrow \infty} x^{k}=\hat{x}$.

Case 2: Suppose that $\lim _{k \rightarrow \infty} \eta_{k}=0$. Let $(\bar{x}, \bar{\xi})$ be any cluster point of $\left\{\left(x^{k}, \xi^{k}\right)\right\}$. Then there exists some subsequence $\left\{\left(x^{k_{i}}, \xi^{k_{i}}\right)\right\}$ converging to $(\bar{x}, \bar{\xi})$. Since $F$ is upper semicontinuous with compact values, it follows from Proposition 3.7 of [1] that $F$ is closed, and so $\bar{\xi} \in F(\bar{x})$. By the choice of $\eta_{k},(3.1)$ implies that

$$
\inf _{y \in F\left(x^{k_{i}}-\gamma^{-1} \eta_{k_{i}} r_{\mu}\left(x^{\left.\left.k_{i}, \xi^{k_{i}}\right)\right)}\right.\right.}\left\langle\xi^{k_{i}}-y, r_{\mu}\left(x^{k_{i}}, \xi^{k_{i}}\right)\right\rangle>\sigma\left\|r_{\mu}\left(x^{k_{i}}, \xi^{k_{i}}\right)\right\|^{2}, \quad \forall i .
$$


Taking into account the boundedness of $\left\{r_{\mu}\left(x^{k_{i}}, \xi^{k_{i}}\right)\right\}$ and $\lim _{i \rightarrow \infty} \eta_{k_{i}}=0$, we have

$$
\lim _{i \rightarrow \infty} \eta_{k_{i}} r_{\mu}\left(x^{k_{i}}, \xi^{k_{i}}\right)=0 \text {. }
$$

Since $F$ and $r_{\mu}(\cdot, \cdot)$ are continuous, it follows from (4.7) that

$$
\sigma\left\|r_{\mu}(\bar{x}, \bar{\xi})\right\|^{2} \leqslant \inf _{y \in F(\bar{x})}\left\langle\bar{\xi}-y, r_{\mu}(\bar{x}, \bar{\xi})\right\rangle \text {. }
$$

Combining $\bar{\xi} \in \mathrm{F}(\bar{x})$, we get

$$
\inf _{y \in F(\bar{x})}\left\langle\bar{\xi}-y, r_{\mu}(\bar{x}, \bar{\xi})\right\rangle \leqslant\left\langle\bar{\xi}-\bar{\xi}, r_{\mu}(\bar{x}, \bar{\xi})\right\rangle=0 .
$$

This, together with (4.8) and $\sigma>0$, implies that $r_{\mu}(\bar{x}, \bar{\xi})=0$. Applying the similar argument in Case 1, we get that $\lim _{k \rightarrow \infty} x^{k}=\bar{x}$ and $\bar{x} \in \operatorname{SOL}(F, C)$. This completes the proof.

Remark 4.5. Compared with the works of $[2,11]$, since condition (A3) is not easy to check, it is often replaced by the pseudomonotonicity of the mapping when we use it. In order to support our results, we should provide an example, where the mapping F satisfies assumptions (H1) and (H2), but it is not pseudomonotone.

Example 4.6. Let $\mathrm{a} \in \mathbb{R}_{+}, \mathrm{C}=[0, \mathrm{a}] \times\{0\} \times[0, \mathrm{a}] \in \mathbb{R}^{3}$. Define $\mathrm{F}: \mathrm{C} \rightrightarrows \mathbb{R}^{3}$ as follows:

$$
\mathrm{F}(x):=\mathrm{F}\left(\mathrm{x}_{1}, 0, x_{3}\right)=\left\{\left(\xi_{1}, \xi_{2}, 0\right) \in \mathbb{R}^{3}:-a \leqslant \xi_{1} \leqslant-x_{1},-a \leqslant \xi_{2} \leqslant a\right\} .
$$

Then one has

(i) the mapping $\mathrm{F}$ is continuous with nonempty and compact values;

(ii) the mapping $\mathrm{F}$ is semistrictly quasimonotone;

(iii) the mapping $\mathrm{F}$ is not pseudomonotone;

(iv) $\operatorname{SOL}_{\mathrm{D}}(\mathrm{F}, \mathrm{C}) \neq \emptyset$.

Proof.

(i) First, we show that $F$ is upper semicontinuous. Indeed, for every $x=\left(x_{1}, 0, x_{3}\right) \in C$, and for any $\varepsilon>0$, denote

$$
V=\left\{\left(\xi_{1}, \xi_{2}, \xi_{3}\right) \in \mathbb{R}^{3}:-a-\varepsilon<\xi_{1}<-x_{1}+\varepsilon,-a-\varepsilon<\xi_{1}<a+\varepsilon,-\varepsilon<\xi_{3}<\varepsilon\right\},
$$

which is an open set containing $F(x)$. Take $U=O\left(x ; \frac{\varepsilon}{2}\right)$, an open ball centered at $x$ and radius $\frac{\varepsilon}{2}$. Then for any $y=\left(y_{1}, 0, y_{3}\right) \in U \cap C$, we have $\left|x_{1}-y_{1}\right|<\frac{\varepsilon}{2}$, i.e., $-x_{1}-\frac{\varepsilon}{2}<-y_{1}<-x_{1}+\frac{\varepsilon}{2}$. So,

$$
\begin{aligned}
\mathrm{F}\left(\mathrm{y}_{1}, 0, \mathrm{y}_{3}\right) & =\left\{\left(\xi_{1}, \xi_{2}, 0\right) \in \mathbb{R}^{3}:-a \leqslant \xi_{1} \leqslant-y_{1},-a \leqslant \xi_{2} \leqslant a\right\} \\
& \subseteq\left\{\left(\xi_{1}, \xi_{2}, 0\right) \in \mathbb{R}^{3}:-a \leqslant \xi_{1} \leqslant-x_{1}+\frac{\varepsilon}{2},-a \leqslant \xi_{2} \leqslant a\right\} \subseteq V .
\end{aligned}
$$

Hence, by arbitrariness of $x \in C$, we have that $F$ is upper semicontinuous.

Secondly, we show that $F$ is lower semicontinuous. Indeed, for every $x=\left(x_{1}, 0, x_{3}\right) \in C$, if given any sequence $\left\{x^{k}\right\} \subset C$ converging to $x$, and any $\xi \in F(x)$, by the continuity of the function $f_{1}(x)=-x_{1}$, which is a constrained condition of $F(x)$, we conclude that there exists a sequence $\left\{\xi^{k}\right\}$ with $\xi^{k} \in F\left(x^{k}\right)$ converging to $\xi$. Hence, by arbitrariness of $x \in C$, we have that $F$ is lower semicontinuous.

Moreover, it is obvious that $F$ has nonempty and compact values.

(ii) Let $x=\left(x_{1}, 0, x_{3}\right), y=\left(y_{1}, 0, y_{3}\right) \in C$, and $\xi \in F(x)$ satisfy $\langle\xi, y-x\rangle>0$. Then we have

$$
\langle\xi, y-x\rangle=\left\langle\left(\xi_{1}, \xi_{2}, 0\right),\left(y_{1}-x_{1}, 0, y_{3}-x_{3}\right)\right\rangle=\xi_{1}\left(y_{1}-x_{1}\right)>0,
$$

which implies that $\xi_{1} \neq 0$ and $y_{1}-x_{1}<0$. For any $\eta=\left(\eta_{1}, \eta_{2}, 0\right) \in F(y)$, we have 


$$
\langle\eta, y-x\rangle=\left\langle\left(\eta_{1}, \eta_{2}, 0\right),\left(y_{1}-x_{1}, 0, y_{3}-x_{3}\right)\right\rangle=\eta_{1}\left(y_{1}-x_{1}\right) \geqslant 0
$$

Therefore, $F$ is quasimonotone. Moreover, it follows from $y_{1}<x_{1}$ that $x_{1} \neq 0$ or $y_{1} \neq 0$. Then we can take $z=\left(z_{1}, 0, z_{3}\right) \in\left(\frac{y+x}{2}, y\right)$ such that $z_{1} \neq 0$. Thus, for any $\zeta=\left(\zeta_{1}, \zeta_{2}, 0\right) \in \mathrm{F}(z)$, we have $\zeta_{1} \neq 0$, and so

$$
\langle\zeta, y-x\rangle=\left\langle\left(\zeta_{1}, \zeta_{2}, 0\right),\left(y_{1}-x_{1}, 0, y_{3}-x_{3}\right)\right\rangle=\zeta_{1}\left(y_{1}-x_{1}\right)>0
$$

Therefore, $\mathrm{F}$ is semistrictly quasimonotone.

(iii) Select $x^{0}=(0,0,0), y^{0}=(a, 0,0), \xi^{0}=(0,0,0) \in F\left(x^{0}\right)$, and $\eta^{0}=(-a, a, 0)$. We have

$$
\left\langle\xi^{0}, y^{0}-x^{0}\right\rangle=0, \quad \text { and }\left\langle\eta^{0}, y^{0}-x^{0}\right\rangle=-a^{2}<0 .
$$

This is a contradiction with the definition of pseudomonotone mappings (see item (iii) of Definition 2.1). Therefore, $\mathrm{F}$ is not a pseudomonotone mapping.

(iv) Firstly, it is easy to see that $\left(\frac{a}{2}, 0, \frac{a}{2}\right) \in \operatorname{inn}(C) \neq \emptyset$. Secondly, the upper semicontinuity of $F$ (see item (i) of this theorem) implies its hemicontinuity. Combining item (ii) and applying Theorem 2.9, we conclude that $\mathrm{SOL}_{\mathrm{D}}(\mathrm{F}, \mathrm{C}) \neq \emptyset$. This completes the proof.

In the sequel, in order to show the contribution of this paper from the theoretical viewpoint, we will show that we have removed condition (A3) and the convexity of $F(x)$ for all $x \in C$ in (A2) used in $[2,11]$. Recall the known relationship between $\mathrm{SOL}(\mathrm{F}, \mathrm{C})$ and $\mathrm{SOL}_{\mathrm{D}}(\mathrm{F}, \mathrm{C})$, see, for example, Proposition 2.2 of [9].

Proposition 4.7. Let $\mathrm{C}$ be a nonempty and convex subset of $\mathbb{R}^{\mathrm{n}}$, and let $\mathrm{F}$ be a hemicontinuous mapping with nonempty, compact, and convex values. Then $S O L_{\mathrm{D}}(\mathrm{F}, \mathrm{C}) \subseteq S O L(\mathrm{~F}, \mathrm{C})$.

On the other hand, it is clear that if condition (A3) is true, we have $\operatorname{SOL}(\mathrm{F}, \mathrm{C}) \subseteq \mathrm{SOL}_{\mathrm{D}}(\mathrm{F}, \mathrm{C})$. This together with Proposition 4.7 implies that $\mathrm{SOL}(\mathrm{F}, \mathrm{C})=\mathrm{SOL}_{\mathrm{D}}(\mathrm{F}, \mathrm{C})$ under the assumptions (A1)-(A3). Thus, condition (A1) can be replaced by (H1). Therefore, the condition combinations used in $[2,11]$ become (H1), (A2), and (A3). Compared with the condition combinations (H1) and (H2) used in this paper, it is easy to find that condition (A3) is removed and we do not add other conditions.

\section{Concluding remarks}

In this paper, we suggest a new projection-type method for GVI $(F, C)$. Relying on the dual variational inequality, we prove the convergence of the proposed method. We also give an example to support our results. Condition (A3), widely used in literatures, is removed in our work. Unfortunately, unlike the work of $[2,11]$, it is somewhat difficult to analyze the rate of convergence of the proposed method under the same conditions. As pointed in the last part of Sect. 4, if assumptions (A1)-(A3) are true, then we have $\operatorname{SOL}(\mathrm{F}, \mathrm{C})=\mathrm{SOL}_{\mathrm{D}}(\mathrm{F}, \mathrm{C})$. Thus, as long as the same or stronger assumptions are used, we can assert that the sequence generated by those methods is also convergent to an element of $\mathrm{SOL}_{\mathrm{D}}(\mathrm{F}, \mathrm{C}$ ) (see, for example, $[2,5,11])$. While the absence of $\operatorname{SOL}(F, C)=\operatorname{SOL}_{D}(F, C)$ in this paper, Theorem 4.4 only says that the sequence produced by our algorithm converges to a solution of GVI(F,C). More precisely, now we wonder whether the limit point of the sequence is an element of $\operatorname{SOL}_{D}(F, C)$ or not because of $\mathrm{SOL}_{\mathrm{D}}(\mathrm{F}, \mathrm{C}) \subseteq \mathrm{SOL}(\mathrm{F}, \mathrm{C})$ (see Proposition 4.7). If the answer is affirmative, we can ensure that the rate of convergence of Algorithm 3.1 is sublinear at least by deduction similar to those $[2,5,11,16]$.

\section{Acknowledgment}

This work was supported by National Natural Science Foundations of China (11561008), Guangxi Natural Science Foundation (2014GXNSFAA118006), Key Project of Scientific Research Foundation of Guangxi Department of Education (ZD2014045), Special Fund for Guangxi Bagui Scholars (WBS 2014-04), Xiangsihu Young Scholars and Innovative Research Team of GXUN (Min-Da 2015-13-02) and Graduates Innovative Project of GXUN (gxun-chxs2015092). 


\section{References}

[1] J. P. Aubin, I. Ekeland, Applied nonlinear analysis, Pure and Applied Mathematics (New York), A Wiley-Interscience Publication, John Wiley \& Sons, Inc., New York, (1984). 4, 4

[2] C.-J. Fang, Y.-R. He, A double projection algorithm for multi-valued variational inequalities and a unified framework of the method, Appl. Math. Comput., 217 (2011), 9543-9551. 1, 3.2, 3.3, 4.5, 4, 4, 5

[3] N. Hadjisavvas, S. Schaible, On strong pseudomonotonicity and (semi)strict quasimonotonicity, J. Optim. Theory Appl., 79 (1993), 139-155. 1, 2.2

[4] N. Hadjisavvas, S. Schaible, Quasimonotone variational inequalities in Banach spaces, J. Optim. Theory Appl., 90 (1996), 95-111. 2

[5] Y.-R. He, A new double projection algorithm for variational inequalities, J. Comput. Appl. Math., 185 (2006), $166-173$. $1,2.5,4.2,5$

[6] A. N. Iusem, M. Nasri, Korpelevich's method for variational inequality problems in Banach spaces, J. Global Optim., 50 (2011), 59-76. 1

[7] A. N. Iusem, L. R. L. Pérez, An extragradient-type algorithm for non-smooth variational inequalities, Optimization, 48 (2000), 309-332. 1

[8] A. N. Iusem, B. F. Svaiter, A variant of Korpelevich's method for variational inequalities with a new search strategy, Optimization, 42 (1997), 309-321. 1

[9] I. V. Konnov, On quasimonotone variational inequalities, J. Optim. Theory Appl., 99 (1998), 165-181. 1, 2, 2.2, 2, 4

[10] G. M. Korpelevič, An extragradient method for finding saddle points and for other problems, (Russian) Ékonom. i Mat. Metody, 12 (1976), 747-756. 1

[11] F.-L. Li, Y.-R. He, An algorithm for generalized variational inequality with pseudomonotone mapping, J. Comput. Appl. Math., 228 (2009), 212-218. 1, 2.7, 3.2, 4.1, 4.5, 4, 4, 5

[12] P. Marcotte, D. L. Zhu, A cutting plane method for solving quasimonotone variational inequalities, Comput. Optim. Appl., 20 (2001), 317-324. 1

[13] M. V. Solodov, B. F. Svaiter, A new projection method for variational inequality problems, SIAM J. Control Optim., 37 (1999), 765-766. 1

[14] G.-J. Tang, N.-J. Huang, Korpelevich's method for variational inequality problems on Hadamard manifolds, J. Global Optim., 54 (2012), 493-509. 1

[15] G.-J. Tang, X. Wang, H.-W. Liu, A projection-type method for variational inequalities on Hadamard manifolds and verification of solution existence, Optimization, 64 (2015), 1081-1096.

[16] G.-J. Tang, M. Zhu, H.-W. Liu, A new extragradient-type method for mixed variational inequalities, Oper. Res. Lett., 43 (2015), 567-572. 1, 5

[17] M.-L. Ye, Y.-R. He, A double projection method for solving variational inequalities without monotonicity, Comput. Optim. Appl., 60 (2015), 141-150. 1, 3.2 